\title{
Effects of casting defects, matrix structures and loading conditions on the fatigue strength of ductile irons
}

\author{
Masahiro Endo ${ }^{1,2, \mathrm{a}}$ and Keiji Yanase ${ }^{1,2}$ \\ ${ }^{1}$ Department of Mechanical Engineering, Fukuoka University, Fukuoka, Japan \\ ${ }^{2}$ Institute of Materials Science and Technology, Fukuoka University, Fukuoka, Japan
}

\begin{abstract}
A novel method is presented to estimate the lower bound of the scatter in fatigue limit of ductile iron based upon the information of microstructural inhomogeneities and loading conditions. The predictive capability of the method was verified by comparing to the experimental data obtained by the rotating-bending, torsion and combined tension-torsion fatigue tests for ductile irons with ferritic, pearlitic and bulls-eye (ferritic/pearlitic) microstructures.
\end{abstract}

\section{Introduction}

Fatigue strength of ductile iron is significantly influenced by the microstructural inhomogeneities, i.e., graphite, casting defects, and matrix structures composed of different phases. Laboratory tests with a limited number of small-sized specimens are likely to provide non-conservative fatigue strength in comparison with the actual strength of high-volume commercial products. Correspondingly, the purpose of this study is to propose a practical method that enables one to predict the lower bound of the scatter in fatigue limit in a judicious manner.

\section{A Practical method for predicting lower bound fatigue limit}

A criterion for multiaxial fatigue was proposed on the basis of the assumption that at threshold level, the time-variation in the mode I stress intensity factor of a crack emanating from a defect under multiaxial cyclic loading is equal to that under uniaxial cyclic loading [1,2]. Then, by using the criterion and the $\sqrt{\text { area }}$ parameter model [3], a practical method was presented to estimate the lower bound of the scatter in fatigue limit of ductile irons under combined loading conditions by taking the statistical nature of microstructural inhomogeneites into consideration.

\section{Comparisons of predictions with experimental results}

Rotating-bending, torsion and combined tension-torsion fatigue tests were carried out for ductile irons with ferritic (FCD400), ferritic/pearlitic (FCD600) and pearlitic (FCD700) microstructures (Fig. 1). The effects of phase difference and mean/static stress in combined loading were also investigated.

\footnotetext{
${ }^{\text {a }}$ Corresponding author: endo@fukuoka-u.ac.jp
}

This is an Open Access article distributed under the terms of the Creative Commons Attribution License 4.0, which permits unrestricted use, distribution, and reproduction in any medium, provided the original work is properly cited. 
Figs. 2-4 show the comparisons of predictions and experimental results. The true lower bounds indicated by thick lines were predicted by considering the worst scenario. The predictions are in good agreement with the experimental results.
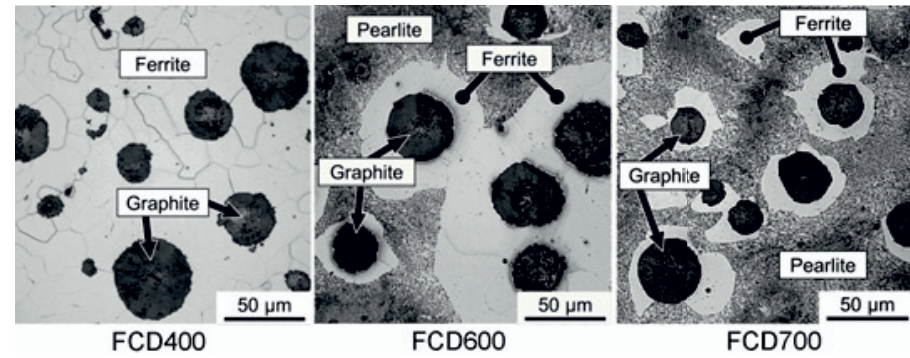

Figure 1. Microstructures of investigated ductile irons.

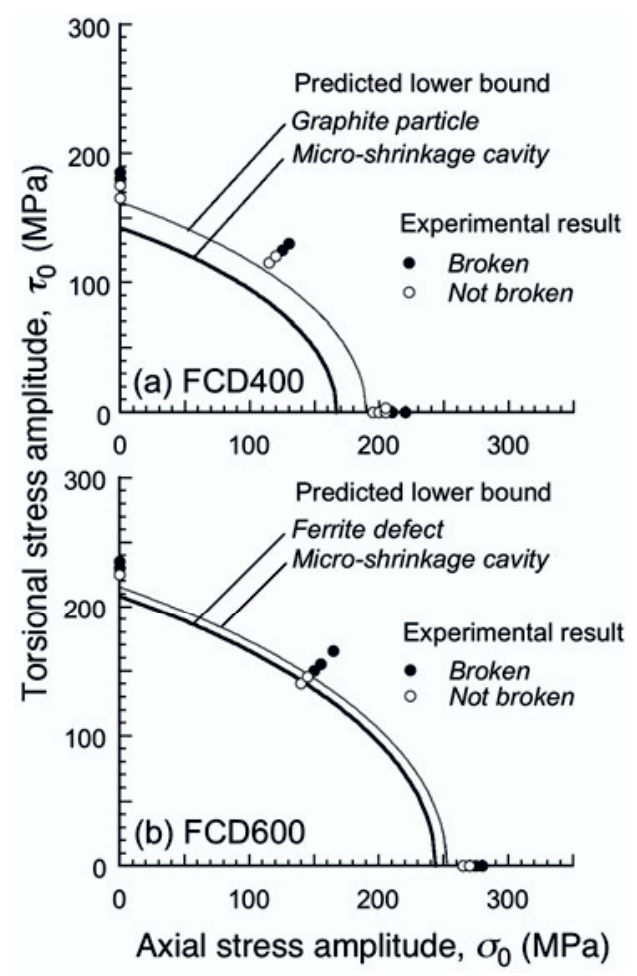

Figure 2. Comparison of predicted lower bound fatigue limits with experimental results (in-phase, zero mean load).

\section{References}

1. M. Endo and I. Ishimoto, Int. J. Fatigue 28, pp. $592-597$ (2006)

2. M. Endo and I. Ishimoto, J. Solid Mechanics and Materials Engineering 1-3, pp. 343-354 (2007)

3. Y. Murakami, Metal Fatigue: Effects of Small Defects and Nonmetallic Inclusions, Elsevier (2002)

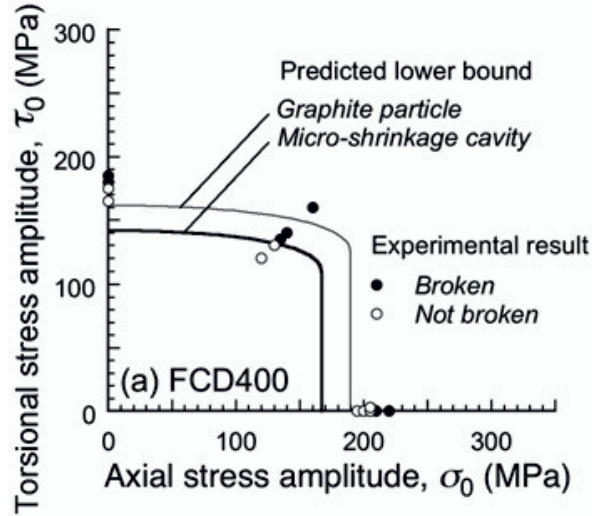

Figure 3. Comparison of predicted lower bound fatigue limits with experimental results ( $90^{\circ}$ out-of-phase, zero mean load).

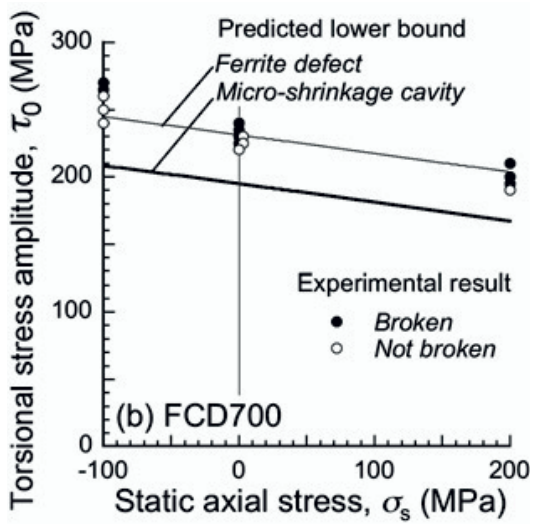

Figure 4. Comparison of predicted lower bound fatigue limits with experimental results (cyclic torsion with static axial load). 